\title{
RACSAM
}

Rev. R. Acad. Cien. Serie A. Mat.

VOL. 102 (1), 2008, pp. 21 38

Geometría y Topología / Geometry and Topology

Artículo panorámico / Survey

\section{Attractors and Inverse Limits}

\section{James Keesling}

\begin{abstract}
This paper surveys some recent results concerning inverse limits of tent maps. The survey concentrates on Ingram's Conjecture. Some motivation is given for the study of such inverse limits.
\end{abstract}

\section{Atractores y límites inversos}

Resumen. Este artículo expone algunos resultados recientes sobre límites inversos de aplicaciones tienda. La exposición se concentra en la Conjetura de Ingram. Se presentan tambien algunas motivaciones para el estudio de tales límites inversos.

\section{Introduction}

For the purposes of this paper a dynamical system is a continuous map $f: X \rightarrow X$ with $X$ a locally compact metric space. If the map $f$ is a homeomorphism, then it can be thought of as a group action of the integers on the space $X, F: \mathbb{Z} \times X \rightarrow X$ with $f(x)=F(1, x)$ for all $x$. We will have occasion to mention continuous dynamical systems. These are flows, continuous maps of $\mathbb{R} \times X$ to $X, F: \mathbb{R} \times X \rightarrow X$, satisfying (1) $F(0, x)=x$ for all $x$ and (2) $F(a+b, x)=F(a, F(b, x))$ for all $a, b \in \mathbb{R}$ and all $x \in X$. A flow is a group action of the additive reals on $X$. Differential equations give rise to flows. Physical systems are typically described this way. However, many systems are described more easily by a continuous function with discrete time.

Dynamical systems are an abstract way of studying the evolution through time of a system. The system could be physical, chemical, or biological. It could also be a model of some human activity such as a business or economy.

A set $A \subset X$ is said to be an attractor if there is an open set $A \subset U \subset X$ having compact closure such that $f(\bar{U}) \subset U$ and $\bigcap_{n=1}^{\infty} f^{n}(\bar{U})=A$. The most obvious attractors are attracting fixed points. However, since the 1960's it has become clear that there are attractors $A$ that have complicated topology with the dynamics of $\left.f\right|_{A}$ being chaotic. This has sparked considerable interest in these strange attractors.

Perhaps the most famous attractor is the one discovered by Edward Lorenz. It has probably had the greatest influence in bringing dynamical systems and attractors to the public consciousness. In the early 1960's Lorenz was a meteorologist studying atmospheric convection at M.I.T. In 1963 [31] he described his remarkable discovery of unexpected behavior of a solution of differential equations modeling this phenomenon. He observed that the resulting flow had deterministic nonperiodic flow. His results provoked wide interest. Among mathematicians there was an attempt to understand the phenomenon that Lorenz

Presentado por José María Montesinos.

Recibido: 23 de septiembre de 2007. Aceptado: 5 de diciembre de 2007.

Palabras clave / Keywords: dynamical system, attractor, inverse limit, tent maps, Ingram's Conjecture, continuum.

Mathematics Subject Classifications: 37E05, 37E10, 37B45

(C) 2008 Real Academia de Ciencias, España. 


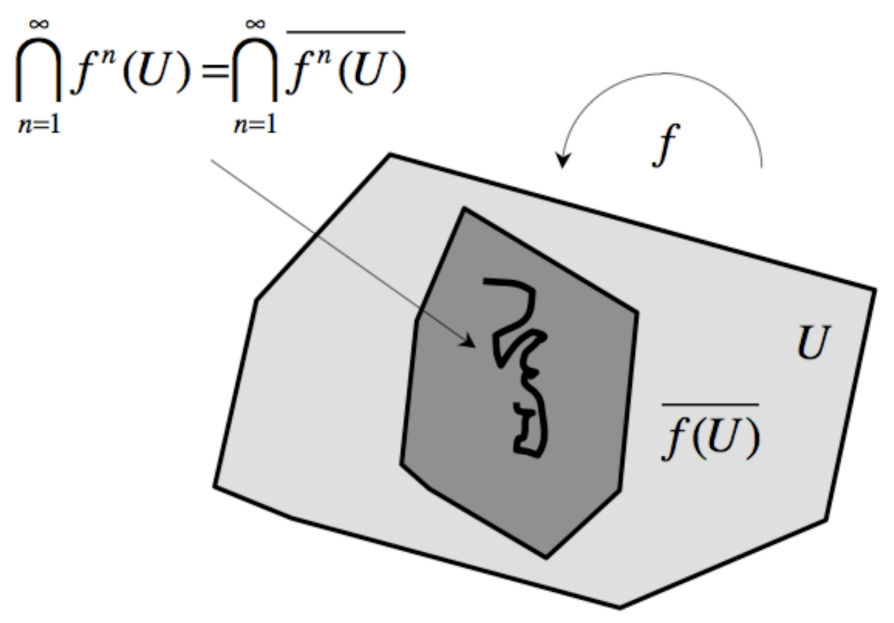

Figure 1. Attractor.

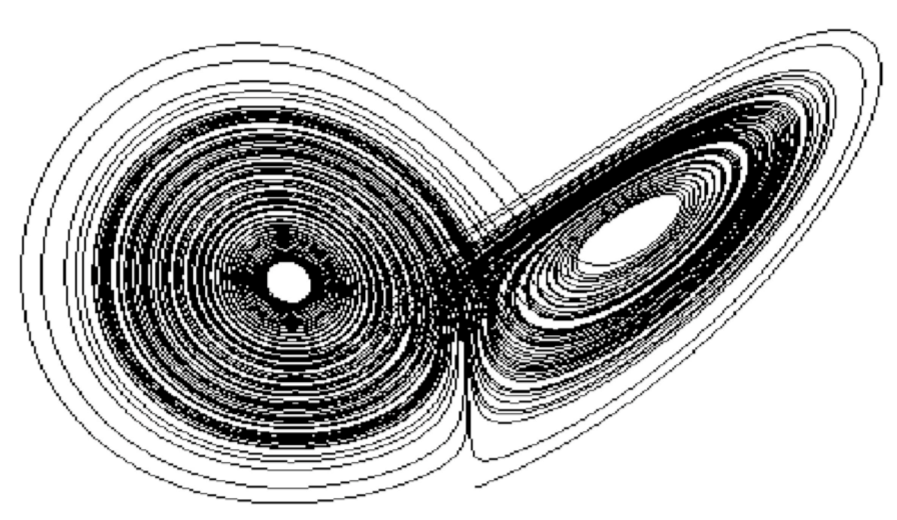

Figure 2. Lorenz attractor.

had observed. It led to an attempt to understand turbulence in terms of the newly founded dynamical systems ([35] and [44]). The Lorenz attractor is an attractor in the sense we have stated at the beginning of this section. It has an attracting neighborhood that is an ellipsoid. The ellipsoid maps into itself under the flow induced by the system of differential equations defining model. The intersection of the forward images of this solid ellipsoid forms the attractor.

In addition to studying the physical significance of the Lorenz attractor, there arose the problem of whether it could be analyzed and characterized with full mathematical rigor. Flows based on geometric models were created by mathematicians. These were easier to analyze and seemed to exhibit the behavior of the Lorenz attractor. See the papers by Guckenheimer and Williams ([18] and [19]) for one example. A complete satisfactory analysis of the Lorenz attractor was only recently produced by Warwick Tucker ([39] and [40]).

Around the same time as the discovery of Lorenz, other papers seemed to observe chaotic behavior of maps. The paper by Hénon and Pomeau [21] describes a map related to the return map of the Lorenz flow that seemed to exhibit chaotic behavior. This Hénon attractor is also an attractor in the sense stated at the beginning. However, the conclusions drawn in [21] were drawn largely from computer simulations. 


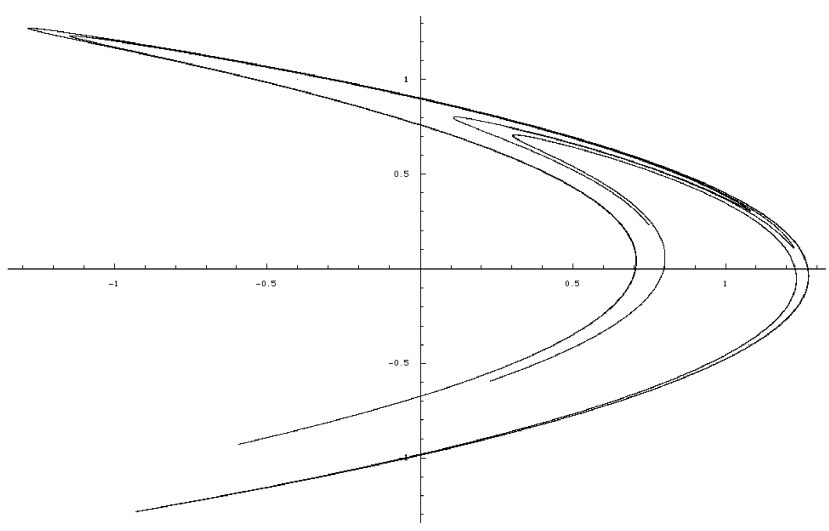

Figure 3. Hénon attractor.

The review by John Guckenheimer of this article in the Mathematical Reviews brings out the difficulty that mathematicians were having at this early stage distinguishing between what was "known" by careful definition and proof and what was "observed" by numerical simulation. That difficulty still continues.

Since the discovery of these examples, many more have been studied. A search of the Internet will yield many geometrically intriguing and mathematically challenging examples. It would take volumes to cover the full scope of the subject.

The study of attractors is closely related to the study of inverse limits. Robert Williams showed that if $f: \mathbb{R}^{n} \rightarrow \mathbb{R}^{n}$ is hyperbolic and $A$ is an attractor for $f$, then $A$ is the inverse limit of a system $K \stackrel{g}{\leftarrow} K \stackrel{g}{\leftarrow} \ldots$ where $K$ is a branched manifold and $g$ is an immersion ([41, 42, 43]). These results were motivated by the work of Steven Smale [37] and his program to understand dynamical systems and hyperbolic attractors.

We use the notation $A=(K, g)$ for an inverse limit where the inverse sequence of spaces and maps are all the same. For such an inverse system, there is a shift map $\sigma:(K, g) \rightarrow(K, g)$ defined by $\sigma\left(\left(x_{0}, x_{1}, x_{2}, \ldots\right)\right)=\left(g\left(x_{0}\right), x_{0}, x_{1}, \ldots\right)$. Williams also showed that $\left.f\right|_{A}$ is conjugate to this shift map. See the section on inverse limits for more details.

Williams' classification of hyperbolic attractors has proved very useful. Many conclusions can be drawn about the topology of the attractor and the dynamics of $\left.f\right|_{A}$. For instance, at every point $x \in A, x$ has a neighborhood in $A$ that is homeomorphic to a Cantor set cross $I^{n}$. The periodic points of $\left.f\right|_{A}$ will be dense in $A$. More about the dynamics of $f$ on the limit set can be determined by properties of the shift map mentioned above. From Williams' work it seemed that there might be some hope that the topology of attractors and the dynamics on them might be fully understood. It could be said that, for hyperbolic attractors at least, the problem was solved by Williams' work.

Despite this glowing picture, the classification of attractors is far from complete. There is even difficulty classifying a very simple case. Suppose that we have an attractor represented as an inverse limit of the following form, $A_{s}=\left(I, f_{s}\right)$ where $I$ is the unit interval, $[0,1]$, and $f_{s}: I \rightarrow I$ is defined by

$$
f_{s}(t)= \begin{cases}s \cdot t & \text { if } 0 \leq t \leq \frac{1}{2} \\ s \cdot(1-t) & \text { if } \frac{1}{2} \leq t \leq 1\end{cases}
$$

where $1 \leq s \leq 2$. We will show in the sections that follow that a classification of these inverse limits can be given only in special cases and that many of them have surprising complexity.

From now on the paper will concentrate on inverse limits. The next section of the paper will give precise definitions for those not familiar. The use of inverse limits in the study of attractors has proved extremely valuable. However, inverse limit spaces have many other applications and are of considerable interest to mathematicians as an area of research in themselves. The interest in them is not limited to the study of attractors. 


\section{Inverse Limits}

We will mainly be considering compact metric spaces and inverse sequences in the study of inverse limits. So, let

$$
\left\{X_{i}, f_{i}\right\}=X_{0} \stackrel{f_{0}}{\longleftarrow} X_{1} \stackrel{f_{1}}{\longleftarrow} X_{2} \stackrel{f_{2}}{\longleftarrow} \cdots
$$

be an inverse sequence of compact metric spaces and continuous maps. We define the inverse limit as the subset of a product space.

$$
\lim _{\longleftarrow}\left\{X_{i}, f_{i}\right\}_{i=0}^{\infty}=\left\{\left(x_{i}\right) \in \prod_{i=0}^{\infty} X_{i} \mid x_{i}=f\left(x_{i+1}\right) \text { for all } i\right\}
$$

There are projections from the inverse limit space to each of the spaces in the inverse system. These are just the projections from the product space to each of its factors. We denote these maps by

$$
\pi_{j}: \lim _{\longleftarrow}\left\{X_{i}, f_{i}\right\} \rightarrow X_{j} \quad \text { for } j=0,1,2, \ldots
$$

These inverse systems are easier if the spaces are all the same compact metric space $X$ and the functions are all the same $f$. In this case the inverse limit is denoted by $(X, f)$. In this special case there is a map on the inverse limit space induced by the map $f: X \rightarrow X$. This is the shift map, $\sigma_{f}:(X, f) \rightarrow(X, f)$. It is defined by

$$
\sigma_{f}\left(\left(x_{0}, x_{1}, x_{2}, \ldots\right)\right)=\left(f\left(x_{0}\right), x_{0}, x_{1}, \ldots\right) .
$$

It is the unique map that makes the diagram below commute for all $i=0,1,2, \ldots$

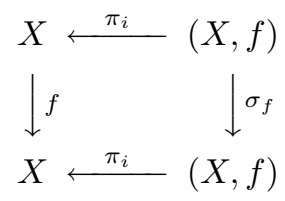

The shift map is a homeomorphism on the limit space. The inverse of the shift is given by

$$
\sigma_{f}^{-1}\left(\left(x_{0}, x_{1}, x_{2}, \ldots\right)\right)=\left(x_{1}, x_{2}, x_{3}, \ldots\right) .
$$

Inverse limits play a vital role in dynamical systems. For a few papers using this approach see [4, 8, 5, 23] as well as the papers by Williams $([41,42,43])$ already mentioned. The sections following give some motivation using examples that are easier understood than the research reported in the later sections of the paper. Some recent results are reported in these sections as well.

\section{The Solenoid}

Solenoids can be represented simply as the inverse limits of inverse systems of the form $\left(\mathbb{S}^{1}, z^{n}\right)$ where we think of the circle as the complex numbers of modulus one in the complex plane. The map $z^{n}: \mathbb{S}^{1} \rightarrow \mathbb{S}^{1}$ takes the complex number $z$ to its $n$-th power. The solenoid described this way will be denoted $\Sigma_{n}=$ $\left(\mathbb{S}^{1}, z^{n}\right)$. It is called the $n$-adic solenoid. Figure 4 is a visualization of the dyadic solenoid. Let $F: T \rightarrow T$ be a continuous map of a solid torus into itself such that $F(T)$ wraps twice around the axis of $T$. Then the dyadic solenoid can be visualized as $\Sigma_{2}=\bigcap_{n=1}^{\infty} F^{n}(T)$. Figure 5 gives a graphic visualization of this [22].

The solenoid is a compact connected topological group. The shift map $\sigma_{n}: \Sigma_{n} \rightarrow \Sigma_{n}$ is a topological group homomorphism and in fact an automorphism since the shift is a homeomorphism. If $n=p$ is a prime number, then the automorphism group of $\Sigma_{p}$ is generated by $\sigma_{p}$ and inversion. In this case, the automorphism group is $\operatorname{Aut}\left(\Sigma_{p}\right) \cong \mathbb{Z} \oplus \mathbb{Z}_{2}$. For general $n$, the prime factorization determines the automorphism group. If $n$ has $k$ prime factors, then the automorphism group is $\operatorname{Aut}\left(\Sigma_{n}\right) \cong \mathbb{Z}^{k} \oplus \mathbb{Z}_{2}$. The shift and inversion do not generate the whole automorphism group in this case. 


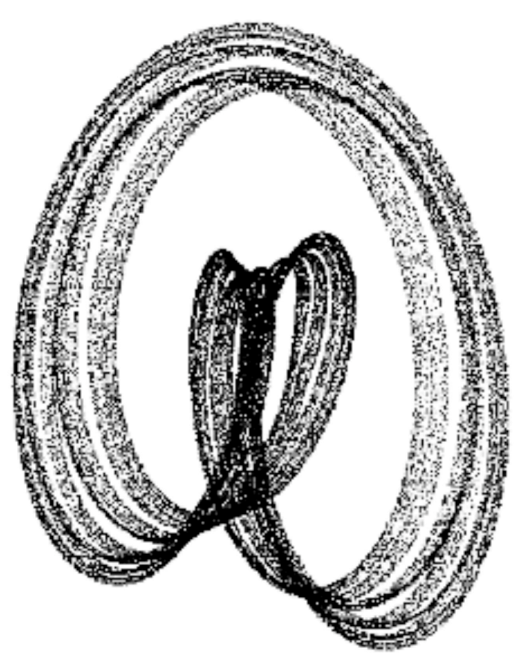

Figure 4. Dyadic solenoid.

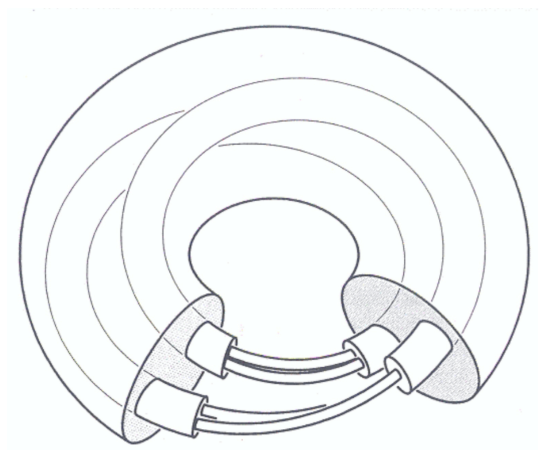

Figure 5. The dyadic solenoid as an attractor [22].

In the general case a solenoid is given as the inverse limit of an inverse system of the following form.

$$
\mathbb{S}^{1} \stackrel{z^{p_{0}}}{\longleftarrow} \mathbb{S}^{1} \stackrel{z^{p_{1}}}{\longleftarrow} \cdots
$$

One can assume without loss of generality that the powers of $z$ are prime numbers. Let $\alpha=\left(p_{0}, p_{1}, \ldots\right)$ be the sequence of prime powers. It is well-known that two solenoids, $\Sigma_{\alpha}$ and $\Sigma_{\beta}$, are homeomorphic if and only if finitely many primes can be deleted from each of $\alpha$ and $\beta$ to form $\alpha^{\prime}$ and $\beta^{\prime}$, respectively, such that each prime occurs the same number of times in $\alpha^{\prime}$ and $\beta^{\prime}$. This result is due to Bing [9] and McCord [32].

A solenoid is an attractor for some continuous $f$ in some $\mathbb{R}^{n}, n \geq 3$, if and only if it is of the form $\Sigma_{k}=\left(\mathbb{S}^{1}, z^{k}\right)$ for some $k>1$. No solenoid can be the attractor of a flow by the result of Günther and Segal [20]. For each point in the solenoid, there is a neighborhood that is homeomorphic to a Cantor set cross an interval.

For more on the structure of solenoids and their homeomorphism groups see Aarts and Fokkink [2] and Keesling [28].

There are recent results concerning the dynamics on general solenoids. Each homeomorphism $h$ of a solenoid $\Sigma_{\alpha}$ has the same topological entropy as a certain automorphism $\alpha \in \operatorname{Aut}\left(\Sigma_{\alpha}\right)$ associated uniquely with $h$. This result was recently proved by Kwapisz [30]. The topological entropy of automorphisms of 


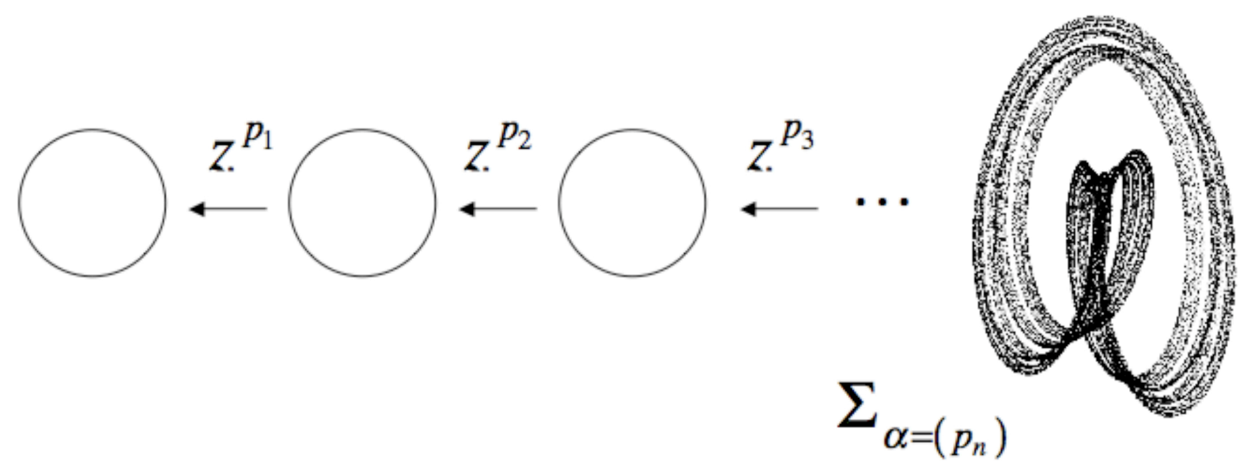

Figure 6. The solenoid as an inverse limit.

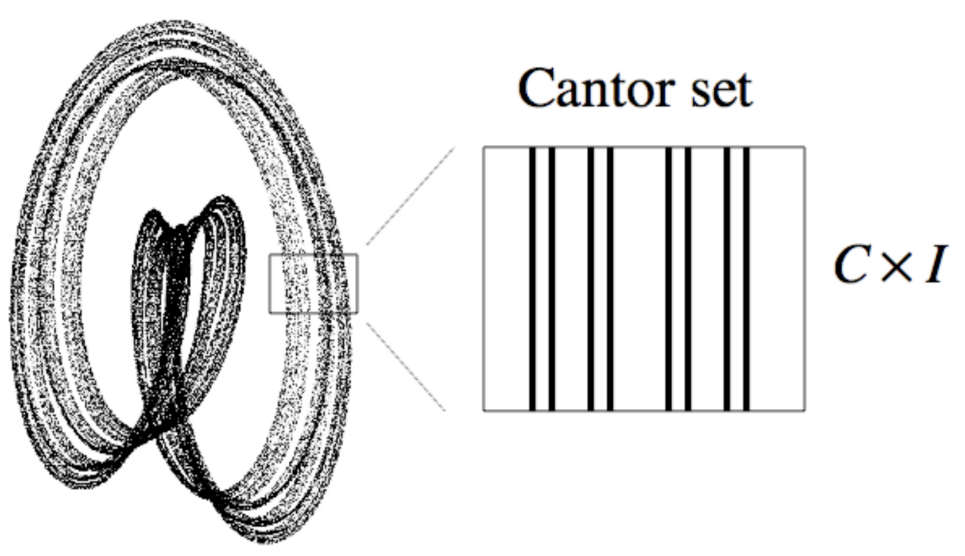

Figure 7. The local structure of the solenoid.

solenoids can be calculated using techniques determined by Abramov [1]. The topological entropy of automorphisms of general compact connected Abelian topological groups has been determined by Juzvinsky [25]. The topological entropy of an autohomeomorphism of these may not be the same as the automorphism to which it is associated by homotopy. So, solenoids are a special case.

\section{The Knaster continuum}

Solenoids were the main motivation for the study of expanding hyperbolic attractors. The work of Williams was in response to questions posed by Smale and his program of analyzing the structure of structurally stable dynamical systems [37]. His work determined the structure of expanding hyperbolic attractors. However, there are many attractors that do not fit into the category he studied. The simplest of these is the Knaster continuum. Let $n \geq 2$ be an integer. Define $K_{n}=\left(I, f_{n}\right)$ where $f_{n}: I \rightarrow I$ is defined by

$$
f_{n}(t)=\left\{\begin{array}{cc}
n \cdot t & 0 \leq t \leq 1 / n \\
2-n \cdot t & 1 / n \leq t \leq 2 / n \\
n \cdot t-2 & 2 / n \leq t \leq 3 / n \\
4-n \cdot t & 3 / n \leq t \leq 4 / n \\
\cdots & \cdots
\end{array}\right.
$$




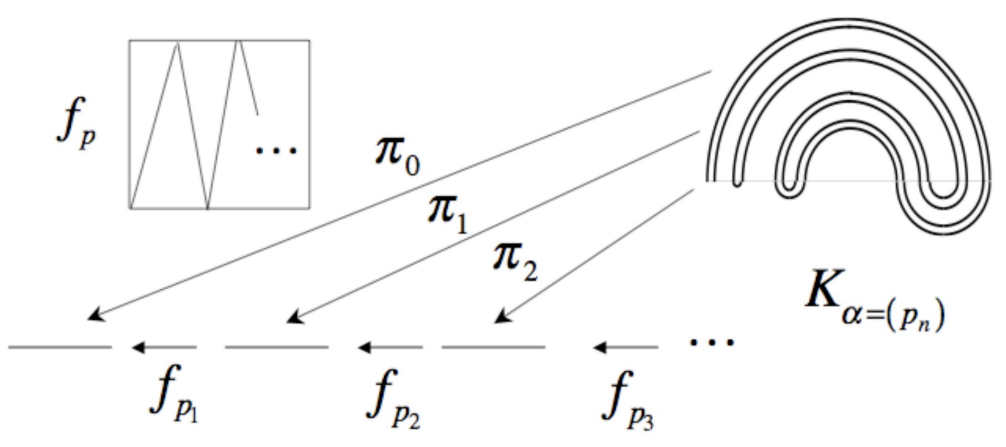

Figure 8. The Knaster continuum as an inverse limit.

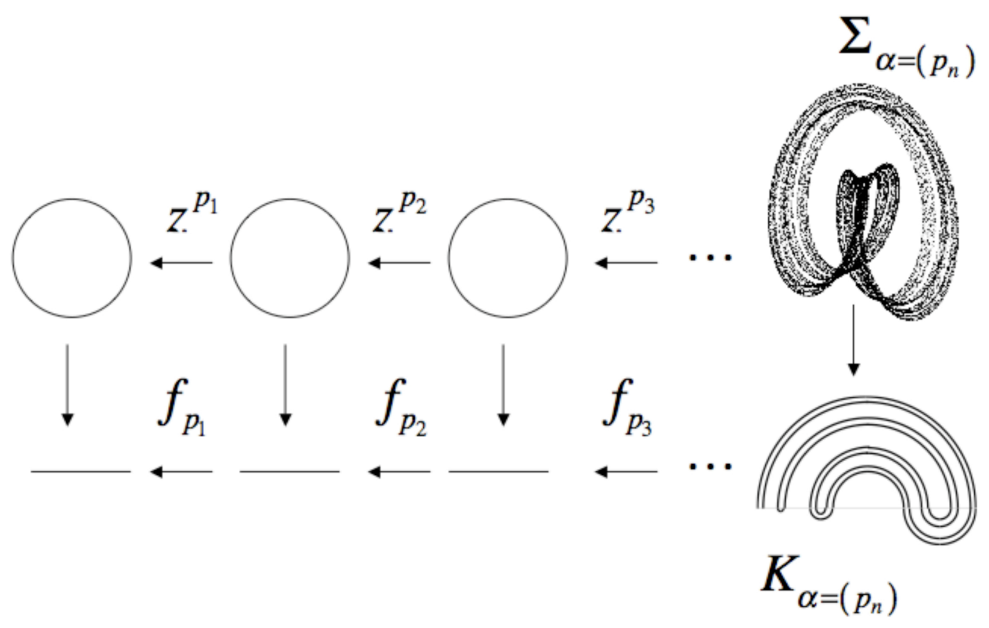

Figure 9. The Knaster continuum as a quotient of a solenoid.

The space $K_{n}$ can also be obtained from the solenoid $\Sigma_{n}$ as a quotient space. Define $x \sim x^{-1}$ on $\Sigma_{n}$ using the topological group structure of $\Sigma_{n}$. Then $K_{n}$ is homeomorphic to $\Sigma_{n} / \sim$. There are generalized Knaster continua. These are similar to the generalized solenoids. A generalized Knaster continuum can be defined by a sequence of primes $\alpha=\left(p_{0}, p_{1}, p_{2}, \ldots\right)$ as $K_{\alpha} \approx \Sigma_{\alpha} / \sim$ where the equivalence relation is $x \sim x^{-1}$. The quotient mapping $q_{\alpha}: \Sigma_{\alpha} \rightarrow K_{\alpha}$ is exactly two-to-one except at one or two points. These generalized Knaster continua can also be represented as inverse limits, $K_{\alpha}=\varliminf_{\lfloor}\left\{I, f_{p_{i}}\right\}$.

Let $a: \Sigma_{\alpha} \rightarrow \Sigma_{\alpha}$ be an automorphism. Then $a\left(x^{-1}\right)=a(x)^{-1}$. Consequently there is a unique homeomorphism $h_{a}: K_{\alpha} \rightarrow K_{\alpha}$ which is the quotient of $a$. Call these homeomorphisms $h_{a}$ on $K_{\alpha}$ automorphisms and denote the set of them by $\operatorname{Aut}\left(K_{\alpha}\right)$.

The identity element $e \in \Sigma_{\alpha}$ has the property that $e=e^{-1}$. Its image under $x_{0}=q_{\alpha}(e)$ is an endpoint in $K_{\alpha}$. That is, if $A$ and $B$ are subcontinua of $K_{\alpha}$ containing $x_{0}$, then either $A \subset B$ or $B \subset A$. There may be another endpoint in $K_{\alpha}$. It depends on whether the sequence $w=(-1,-1,-1, \ldots)$ is a thread in $\Sigma_{\alpha}$. If it is, then $x_{1}=q_{\alpha}(w)$ is also an endpoint. The sequence $w$ is a thread if and only if 2 does not occur in $\alpha$. If $\alpha^{\prime}$ is obtained from $\alpha$ by eliminating finitely many terms, then $\Sigma_{\alpha} \cong \Sigma_{\alpha^{\prime}}$. So, in fact, $K_{\alpha}$ has two endpoints if 2 occurs in $\alpha$ just finitely many times and one endpoint if 2 occurs in $\alpha$ infinitely often.

There are no other endpoints in $K_{\alpha}$. In fact, at every other point $x \in K_{\alpha}$, there is a neighborhood of $x$ 
homeomorphic to a Cantor set cross an interval, $C \times I$. Since $x$ is in the interior of one of the intervals in this product, it cannot be an endpoint.

If $K_{\alpha}$ has just one endpoint, then it must be a fixed point for any homeomorphism of $K_{\alpha}$. If there are two endpoints in $K_{\alpha}$ and $h$ is a homeomorphism, then the two endpoints could possibly be interchanged by $h$. However, $h^{2}$ must leave both endpoints fixed and so must have at least two fixed points. In fact $h^{2 n}$ may have many more fixed points. A study of the fixed points for homeomorphisms of $K_{\alpha}$ has been done by Keesling and Ssembatya [29]. It generalized the work of Aarts and Fokkink [3] that showed that every homeomorphism of $K_{2}$ has at least two fixed points.

If there are $k$ primes that occur infinitely often in the sequence $\alpha$ and 2 is one of these primes, then $\operatorname{Aut}\left(K_{\alpha}\right) \cong \oplus_{n=1}^{k} \mathbb{Z}$. If 2 does not occur infinitely often in $\alpha$, then we need to add another involution to the automorphisms for completeness giving $\operatorname{Aut}\left(K_{\alpha}\right) \cong \oplus_{n=1}^{k} \mathbb{Z} \times \mathbb{Z}_{2}$. The extra involution is generated by the following diagram.

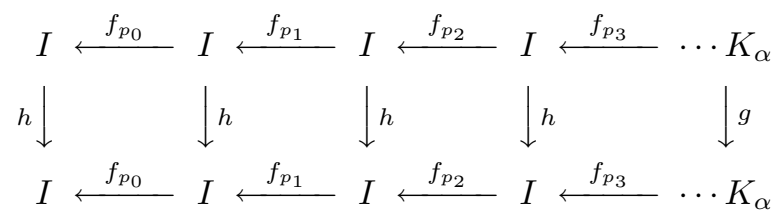

The map $h$ is defined by $h(t)=1-t: I \rightarrow I$. The map $g$ is well-defined provided 2 does not occur as one of the primes. By (1) below, if 2 occurs only finitely many times, then there is a sequence $\beta$ in which 2 does not occur such that $K_{\alpha} \approx K_{\beta}$. Use $K_{\beta}$ to define $g$. This automorphism is not a quotient of an automorphism of $\Sigma_{\alpha}$, but is a standard homeomorphism and is needed for a complete analysis of $K_{\alpha}$. So, $\operatorname{Aut}\left(K_{\alpha}\right) \cong \oplus_{n=1}^{k} \mathbb{Z}$ or $\operatorname{Aut}\left(K_{\alpha}\right) \cong \oplus_{n=1}^{k} \mathbb{Z} \oplus \mathbb{Z}_{2}$ depending on whether 2 does or does not occur infinitely often in $\alpha$.

The following additional facts are known about $K_{\alpha}$.

(1). Two Knaster continua, $K_{\alpha}$ and $K_{\beta}$, are homeomorphic if and only if $\Sigma_{\alpha}$ and $\Sigma_{\beta}$ are homeomorphic.

(2). If $h$ is a homeomorphism of $K_{\alpha}$, then $h$ is isotopic to a unique $a \in \operatorname{Aut}\left(K_{\alpha}\right)$.

(3). If $h$ is a homeomorphism of $K_{\alpha}$, then there are exactly two homeomorphisms $h_{1}, h_{2}: \Sigma_{\alpha} \rightarrow \Sigma_{\alpha}$ making the following diagram commute.

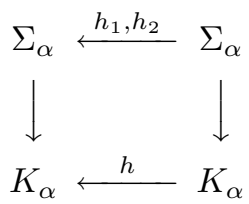

This result was proved by Kwapisz [30]. The result has been accepted and used, but this is the first correct proof in the literature.

(4). Each isotopy between homeomorphisms on $K_{\alpha}$ lifts to two isotopies on $\Sigma_{\alpha}$.

(5). If $h$ is a homeomorphism of $K_{\alpha}$, then the topological entropy of $h$ is the same as the topological entropy of $h_{1}$ and $h_{2}$ where $h_{1}$ and $h_{2}$ are the homeomorphisms of $\Sigma_{\alpha}$ given in (3).

(6). If $h$ is a homeomorphism of $K_{\alpha}$, then the topological entropy of $h$ is the same as the automorphism to which it is isotopic. This is a simple consequence of (2)

(7). If $h$ is any homeomorphism of $K_{2}$, then $h$ has at least two fixed points [3]]. The same is true for any $K_{\alpha}$ where 2 occurs infinitely often and 3 occurs only finitely many times in $\alpha$. For more results of this type see [29] mentioned above. 


\section{Basic properties of inverse limits of tent maps}

From what has been said about solenoids and Knaster continua, one may get the impression that attractors have a simple and easily comprehensible structure. The solenoids are simplest since they are expanding hyperbolic attractors and are understood by Williams' results. The results of Williams were motivated by what was known about solenoids.

The Knaster continua are slightly more complex. At all but one or two points, the local structure is a Cantor set cross an interval, $C \times I$. The one or two exceptional points are endpoints and the local structure is also known in this case.

Consider the sequences of primes $\alpha=\left(p_{0}, p_{1}, \ldots\right)$ and $\beta=\left(q_{0}, q_{1}, \ldots\right)$ defining $\Sigma_{\alpha}$ and $\Sigma_{\beta}$, respectively ( $K_{\alpha}$ and $K_{\beta}$, respectively). A simple comparison of these sequences tells us whether $\Sigma_{\alpha}$ and $\Sigma_{\beta}$ are homeomorphic ( $K_{\alpha}$ and $K_{\beta}$ are homeomorphic). We know the structure of the homeomorphism groups of these spaces. Each homeomorphism of $\Sigma_{\alpha}$ which leaves the identity element fixed is isotopic to a unique automorphism of $\Sigma_{\alpha}$. There is a similar result for Knaster continua.

We now consider inverse limits of tent maps. Tent maps are similar to the maps defining Knaster continua. So, we might expect that these inverse limits would not differ much from Knaster continua. This is not the case. We get spaces having much greater complexity and we are very far from a complete understanding of them.

Let $s \in[1,2]$ and consider the family of maps $f_{s}:[0,1] \rightarrow[0,1]$ defined in the following way.

$$
f_{s}(t)= \begin{cases}s \cdot t & 0 \leq t \leq \frac{1}{2} \\ s \cdot(1-t) & \frac{1}{2} \leq t \leq 1\end{cases}
$$

This is known as the tent family. It arises naturally in the study of the dynamics of unimodal maps. For instance, given any unimodal map $g:[0,1] \rightarrow[0,1]$, there is a unimodal map $f:[0,1] \rightarrow[0,1]$ whose slope is everywhere $\pm s$ and a continuous $h:[0,1] \rightarrow[0,1]$ with $h \circ g=f \circ h$ with the topological entropy of $f$ being the same as the topological entropy of $g$. See Milnor and Thurston [34] for this result and similar results for piecewise monotone functions on an interval.

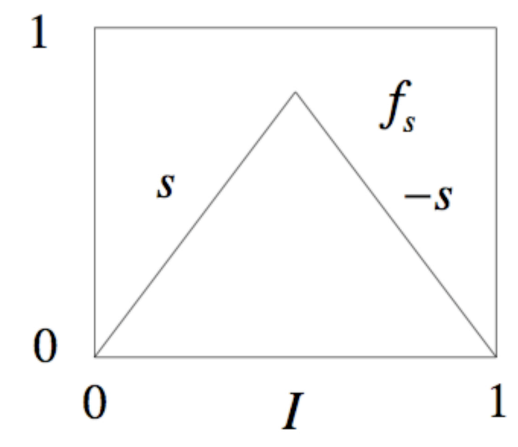

Figure 10. The tent family.

A natural question arises. What is the structure of $\left(I, f_{s}\right)$ ? If we would hope to understand the inverse limits of piecewise monotone maps on an interval, we would need to understand this case.

If $s=1$, then a simple argument shows that $\left(I, f_{s}\right)$ is homeomorphic to an interval. If $s=2$, then we get the Knaster continuum, $K_{2}$. If $1<s<2$, then one prominent structure stands out. There is an open ray which is dense in the inverse limit. Let us denote that ray by $A_{0}$. It is convenient to denote the images of $c$ in the following way $c_{0}=c, c_{1}=f_{s}(c), c_{2}=f_{s}^{2}(c), \ldots$ The first four of these are particularly useful in understanding the inverse limit space under consideration. Figures 11 and 12 help visualize features of the graph of $f_{s}$. 


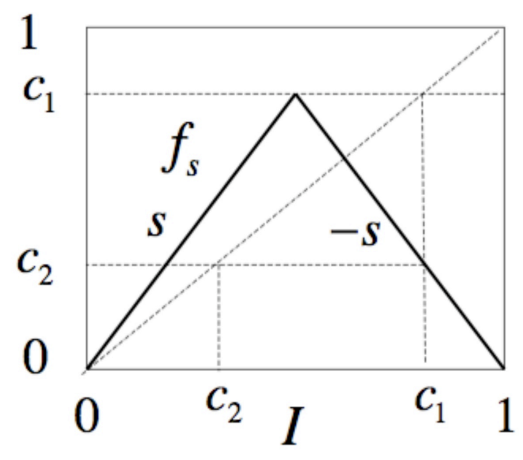

Figure 11. $f_{s}^{-1}\left(\left[0, c_{2}\right)\right) \subset\left[0, c_{2}\right)$

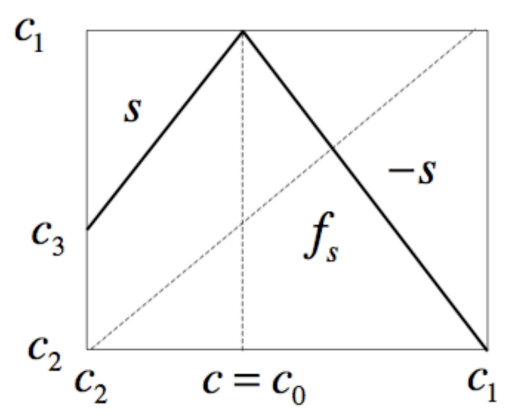

Figure 12. The core.

Consider the interval $\left[0, c_{2}\right)$. Each point in this interval has a unique point in the interval $[0,1]$ which maps to it and that point is in the interval $\left[0, c_{2}\right)$. So we have an inverse system.

$$
\left[0, c_{2}\right) \stackrel{f_{2}}{\longleftarrow}\left[0, c_{2}\right) \longleftarrow \cdots
$$

This forms an open arc in the limit space $\left(I, f_{s}\right)$. This arc is just $\pi_{0}^{-1}\left(\left[0, c_{2}\right)\right)$. The ray $A_{0}$ mentioned above is just the union of the monotone increasing sequence of open sets given below.

$$
A_{0}=\bigcup_{n=0}^{\infty} \pi_{n}^{-1}\left(\left[0, c_{2}\right)\right)
$$

One can show that $A_{0}$ is dense in the inverse limit $\left(I, f_{s}\right)$. We can visualize the inverse limit then as in Figure 13 .

Consider the map $f_{s}:\left[c_{2}, c_{1}\right] \rightarrow\left[c_{2}, c_{1}\right]$. The rest of the inverse limit $\left(I, f_{s}\right)$ will be the inverse limit of this map and subinterval. We call this the core and denote it by $X_{s}=\left(\left[c_{2}, c_{1}\right], f_{s}\right)$. Most of the study of the space $\left(I, f_{s}\right)$ has concentrated on the core.

\section{Ingram's Conjecture}

In 1995 Tom Ingram conjectured [24] that if $\left(I, f_{s}\right)$ is homeomorphic to $\left(I, f_{t}\right)$, then $s=t$. The conjecture may not be original with Ingram, but he was the first having the boldness to put it in print as an important 


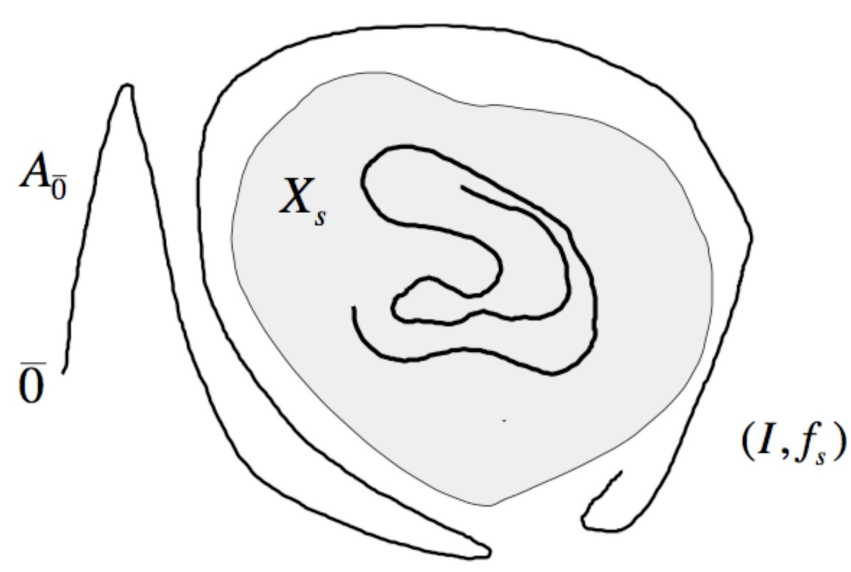

Figure 13. The open ray $A_{0}$ limiting on the core $X_{s}$.

problem to consider. It is the most basic question to ask about these spaces. Much of what we know about $\left(I, f_{s}\right)$ comes from attempting to prove this conjecture.

There is an immediate simplification. If it is true that $X_{s}$ homeomorphic to $X_{t}$ implies that $s=t$, then Ingram's Conjecture is true. Most of the work so far has concentrated on showing this. The open ray $A_{0}$ has largely been ignored and considered superfluous. In Section 9 we will show that $A_{0}$ may actually be a key in solving Ingram's Conjecture. It is certainly a key to understanding the structure of the group of homeomorphisms of $\left(I, f_{s}\right)$.

There are other simplifications used in the pursuit of Ingram's Conjecture. If $\sqrt{2}<s<2$, then $X_{s}$ is indecomposable. A metric $Y$ continuum is indecomposable if whenever $Y$ is the union $Y=A \cup B$, with $A$ and $B$ subcontinua of $Y$, then either $A$ or $B$ is equal to $Y$. This may seem a strange property, but it is invaluable to continuum theorists. The property has been exploited to great advantage. Most papers assume that $\sqrt{2}<s<2$ and consequently that $X_{s}$ is indecomposable. However, it is well known by researchers in the area that if Ingram's Conjecture holds in this case, then it is true for all $1 \leq s \leq 2$. In the next paragraph we give an outline of why this is true.

If $\sqrt[4]{2}<s<\sqrt{2}$, then it can be shown that there is a unique $\sqrt{2}<t<2$ such that there are exactly two copies of $\left(I, f_{t}\right)$ contained in $X_{s}$. These two copies are joined at the endpoints of their respective $A_{0}$ 's and the map $f_{s}$ interchanges these two copies by an map with a fixed point at the point where they are joined. So, if Ingram's Conjecture is true for all $\sqrt{2}<s, t<2$, then it would be true for all $\sqrt[4]{2}<s, t<\sqrt{2}$. In a similar fashion one can in fact show that it is sufficient to show Ingram's Conjecture for $\sqrt{2}<s, t<2$ for it to be true for all $\sqrt[n+1]{2}<s, t<\sqrt[n]{2}$ for $n=1,2,3, \ldots$. This will imply that it is true for all $1 \leq s, t \leq 2$. So, we not only have a range of $s$ for which the core is indecomposable, it is sufficient to concentrate on that range alone to arrive at a complete solution of the problem.

From this point on, we assume that all slopes are in the range, $\sqrt{2}<s<2$.

\section{Periodic turning point and Kailhofer's result}

When Ingram proposed his conjecture, there was perhaps only one result that appeared helpful. It is the following theorem.

Theorem 1 Suppose that $f_{s}: I \rightarrow I$ is a tent map and that $c=\frac{1}{2}$ is periodic of period $n$. Then $X_{s}$ has exactly $n$ endpoints. 
Suppose that $X_{s}$ and $X_{t}$ are homeomorphic for $f_{s}$ and $f_{t}$ tent maps with periodic turning point. Then the theorem above implies that the turning point for $f_{s}$ and $f_{t}$ must have the same period $n$. This looks promising and would be helpful if there were but a small number of slopes $s$ with the turning point having given period. However, this is not the case as can be seen from Table 1 . For period 3 there is just one value of $s$ for which $c$ is period three. For period 5 , there are three values of $s$.

Consider the case of period five. By a very sophisticated argument Marcy Barge and Beverly Diamond showed that for these three cases Ingram's Conjecture holds [5]. Henk Bruin showed Ingram's Conjecture for other special cases when $c$ is periodic [16].

The proof of Barge and Diamond did not hold out much hope for proving Ingram's Conjecture for higher periods. It was algebraic and made use of Maple to make some difficult calculations. The task was made all the more daunting when one realizes how many values of $s$ yield $c$ with the same period as indicated by Table 1

\begin{tabular}{|r|r|}
\hline$n$ & Number of $s$ \\
\hline 3 & 1 \\
\hline 5 & 3 \\
\hline 7 & 9 \\
\hline 11 & 93 \\
\hline 13 & 315 \\
\hline 17 & 3,855 \\
\hline 19 & 13,797 \\
\hline 23 & 182,361 \\
\hline 29 & $9,256,395$ \\
\hline 31 & $34,636,833$ \\
\hline
\end{tabular}

Table 1. Prime periods $n$ and number of $s$ with orbit of $c$ having this period.

One was not likely to solve the problem even for a few values of $n$ by using difficult algebraic calculations. There were just too many cases to contend with. The final breakthrough came in the work of Lois Kailhofer in [26] and [27].

Theorem 2 (Kailhofer [26] and [27]) Suppose that $f_{s}$ and $f_{t}$ are tent maps and the turning point is periodic for both maps. Then if $X_{s}$ is homeomorphic to $X_{t}$, then $s=t$.

This gave great hope for the conjecture by settling a case that had become a main focus of research. The proof was difficult. It was made more difficult by not providing a clear roadmap to the final theorem. A more readable proof has since been published based on the work of Kailhofer, but written so that one could read the paper independently [12]. There are in fact some additional results in this paper. These deal with the structure of the homeomorphism group of the spaces.

Before saying more about the paper [12], let us say some more about the structure of the space $X_{s}$ when $c$ is periodic of period $n$. As was mentioned at the beginning of this section, there are $n$ endpoints in $X_{s}$. These endpoints come from the periodic orbit of $c$. That periodic orbit can be made into $n$ threads that can be shown to be $n$ endpoints in the inverse limit. The other points have neighborhoods homeomorphic to a Cantor set cross an interval.

In the next section we will state what proved to be a valuable insight into the structure of the homeomorphism group. It has suggested an approach that could solve Ingram's Conjecture for all values of $s$.

\section{Homeomorphisms of $X_{s}$ for $c$ periodic}

The paper [12] provides a simplification of Kailhofer's original solution of Ingram's Conjecture for $c$ periodic. It also proves an isotopy result for homeomorphisms of $X_{s}$. The basic results can be stated simply in 


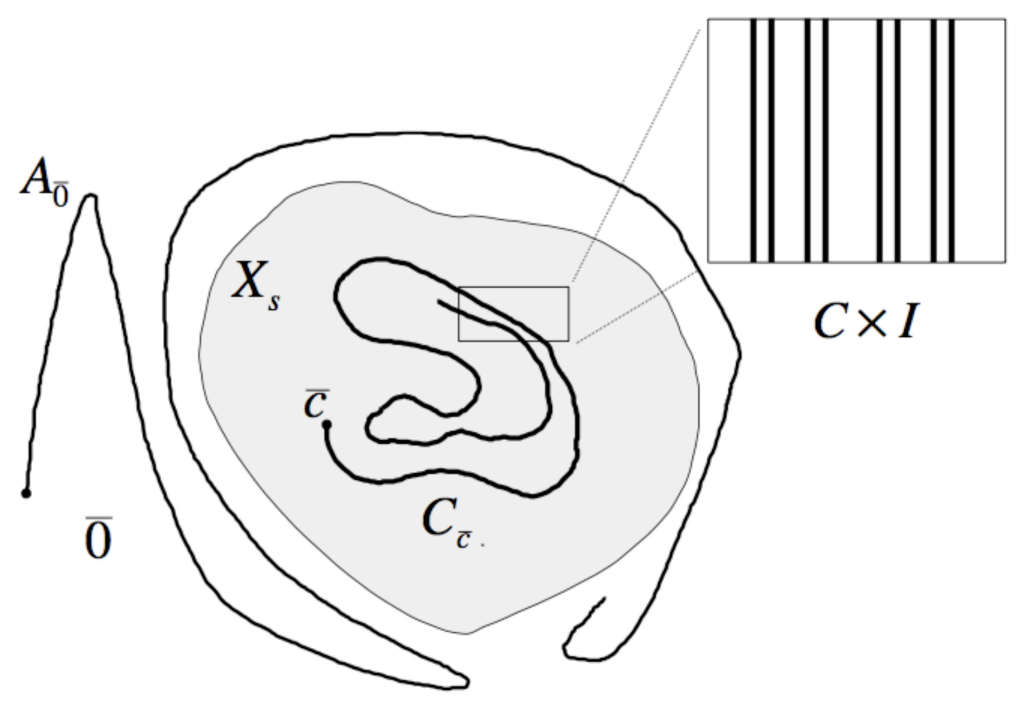

Figure 14. Endpoints and local structure of $X_{s}$ with $c$ periodic.

the form of two theorems.

Theorem 3 ([12]) Suppose that $c$ is period for $f_{s}$. There is an $\varepsilon>0$ such that if $h: X_{s} \rightarrow X_{s}$ is any homeomorphism such that $d(h, \mathrm{id})<\varepsilon$, then $h$ is isotopic to the identity.

Of course, the result implies that if any two homeomorphisms are within $\varepsilon$ of each other then they are isotopic. It is a natural conjecture that every homeomorphism $h: X_{s} \rightarrow X_{s}$ is isotopic to some power of the shift $\sigma$ on $X_{s}$. This is probably true in the case that $c$ is periodic. It is not true for $c$ not periodic. However, in the next section we will show that in the general case it may be true that every homeomorphism $h: X_{s} \rightarrow X_{s}$ is pseudo-isotopic to a power of the shift. What is actually proved in [12] is somewhat less than is believed to be true.

Theorem 4 ([12]) Suppose that $c$ is periodic for $f_{s}$. Let $h: X_{s} \rightarrow X_{s}$ be any homeomorphism. Then there is a positive integer $k$ and an integer $m$ such that $h^{k}$ is isotopic to $\sigma^{m}$.

As stated above, $\sigma$ is just the shift homeomorphism induced by the map $f_{s}$ on the core $X_{s}=\left(\left[c_{2}, c_{1}\right], f_{s}\right)$. The result is reminiscent of what is known about homeomorphisms on Knaster continua. The best result would be if each isotopy class of the homeomorphism group of $X_{s}$ has exactly one power of the shift in it. This would show that the homeomorphism group of $X_{s}$ is very similar to that of the Knaster continua $K_{p}$ for $p$ prime.

Theorem 4 can be used to give a proof of Theorem 2 The idea is to use Theorem 4 to distinguish the two tent maps $f_{s}$ and $f_{t}$ having periodic $c$ with the same period. One can show that if $s<t$, then there is some $k>0$ such that $f_{t}$ has more points of period $k$ than $f_{s}$. On the other hand, by Theorem 4 , one can show that if $X_{s}$ is homeomorphic to $X_{t}$, then for this $k, f_{s}$ and $f_{t}$ have the same number of periodic points, a contradiction.

It would be too complicated to give a satisfactory account of the proof of Theorem 4 . However, the following description together with Figure 15 may give a hint of what is involved. One uses the indecomposability of $X_{s}$. There is a continuous map $\varphi:[0, \infty) \rightarrow X_{s}$ so that $\varphi(0)=\bar{c} \in X_{s}$. The point $\bar{c}$ is the point in the inverse limit that corresponds to the turning point $c$. The trick is to define $\varphi$ carefully in terms of the inverse system so that if $\pi_{n}([\varphi(x), \varphi(y)])$ is one-to-one, then the distance between $x$ and $y,|x-y|$, in $[0, \infty)$ is equal to $s^{n} \cdot\left|\pi_{n}(\varphi(x))-\pi_{n}(\varphi(y))\right|$ in the image of $\pi_{n}$. One can choose $\varepsilon>0$ carefully so that 

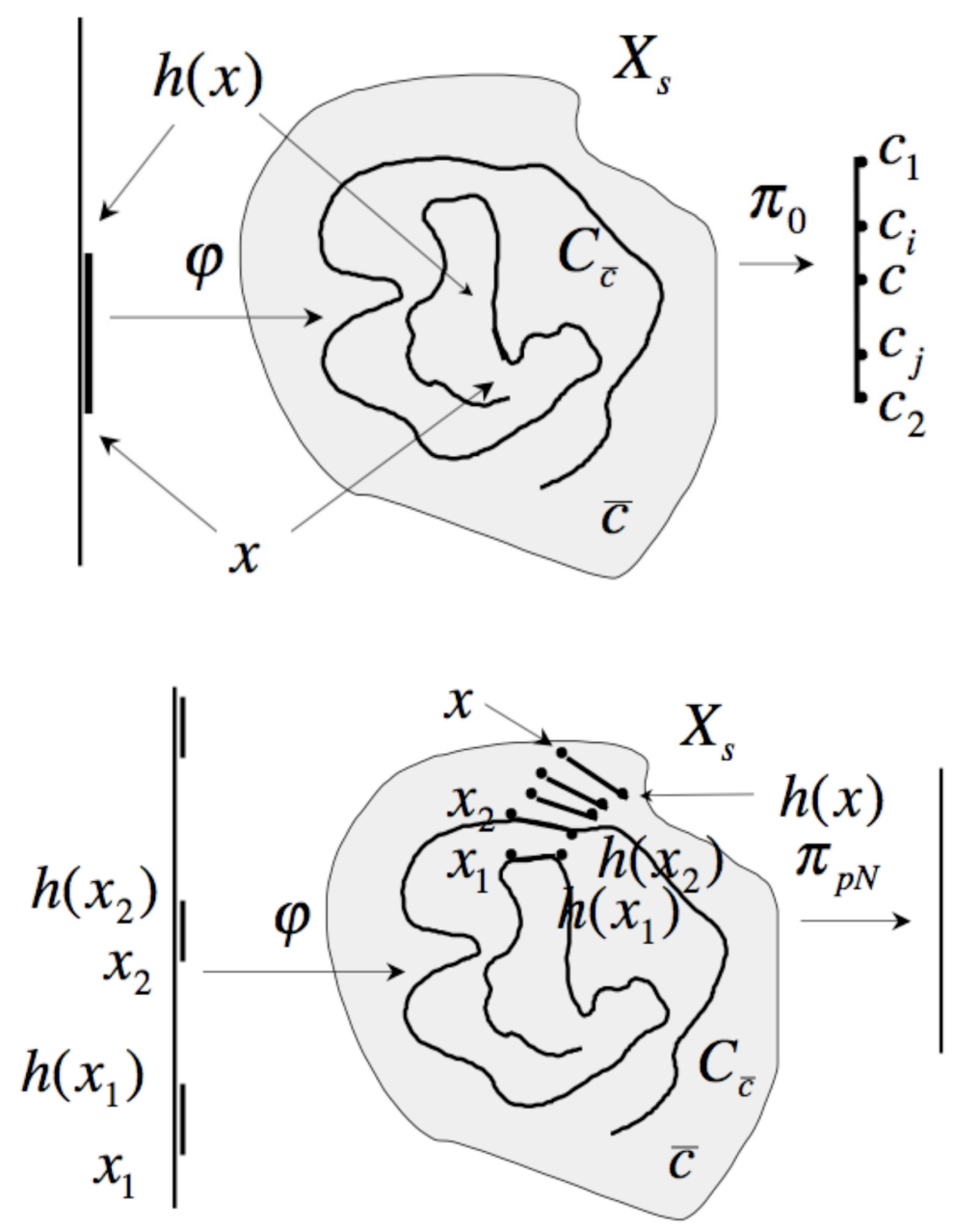

Figure 15. Close homeomorphisms in $X_{s}$ are isotopic.

if $h$ is a homeomorphism of $X_{s}$ which is within $\varepsilon$ of the identity, then $h(\bar{c})=\bar{c}$. Furthermore, the choice of $\varepsilon$ can be made carefully enough that one can show that for all $0<x<\infty,|\bar{h}(x)-x|<M$ for some $M$ where $\bar{h}(x)=\varphi^{-1} \circ h \circ \varphi(x)$. The image of $\varphi, \varphi([0, \infty))$, is dense in $X_{s}$. The bounded distance between $\bar{h}$ and the identity on $[0, \infty)$ can be used to show that for any $x \in X_{s}, x$ and $h(x)$ are connected by an arc in $X_{s}$. One can parameterize these arcs between $x$ and $h(x)$ in such a way as to get an isotopy from the identity to $h$.

\section{A conjecture about homeomorphisms on $\left(I, f_{s}\right)$}

For this section we need the concept of pseudo-isotopic. Suppose that $h_{1}, h_{2}: X_{s} \rightarrow X_{2}$ are homeomorphisms. Suppose that for all $x \in X_{s},\left\{h_{1}(x), h_{2}(x)\right\} \subset B_{x}$, where $B_{x}$ is a proper subcontinuum of $X_{s}$. Then we say that $h_{1}$ and $h_{2}$ are pseudo-isotopic. If $h_{1}$ and $h_{2}$ were homotopic, then $B_{x}$ could be taken to be a Peano continuum. Since Peano continua are arcwise connected, we could in fact take $B_{x}$ to be an arc. If $c$ is periodic, then the only proper subcontinua of $X_{s}$ are points and arcs. This fact was important in the 


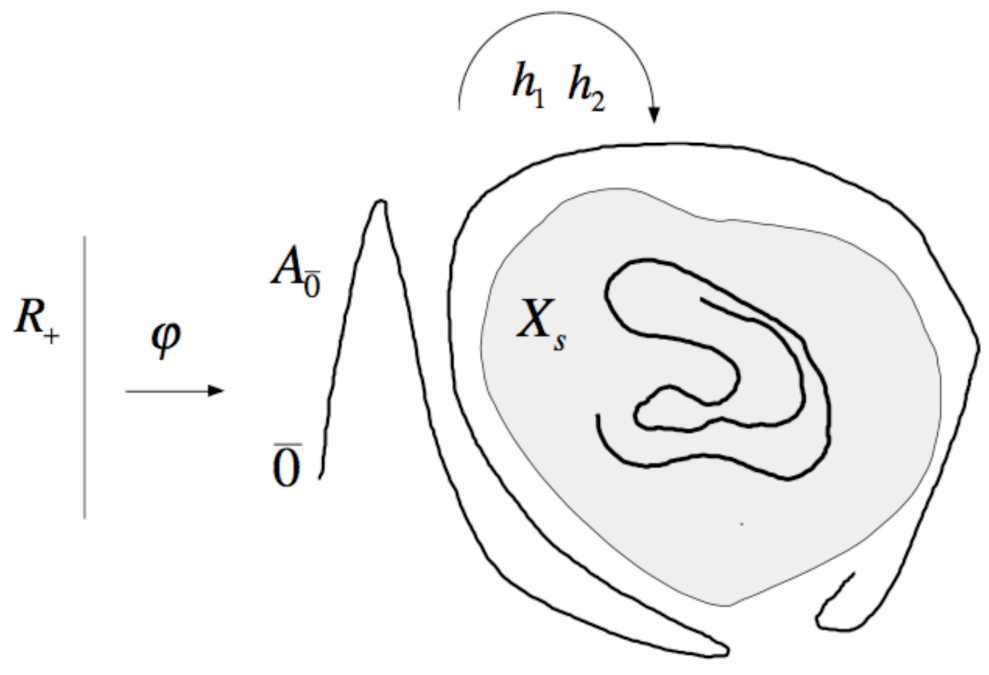

Figure 16. The bounded $\bar{d}$-distance theorem.

proof of Theorem 3 . If $c$ is not periodic, then $X_{s}$ may have many strange subcontinua which are not Peano continua [6].

If $h_{1}$ and $h_{2}$ are pseudo-isotopic on $X_{s}$, then they permute the composants of $X_{s}$ in the same way. That observation is a key element in the proof of the following theorem.

Theorem 5 ([13]) Suppose that $X_{s}$ and $X_{t}$ have the property that if $h$ is a homeomorphism, then there is an integer $m$ such that $h$ is pseudo-isotopic to $\sigma^{m}$. Then if $X_{s}$ and $X_{t}$ are homeomorphic, then $s=t$.

So, a general approach to Ingram's Conjecture would be to show that for every $s$ and every homeomorphism $h$ on $X_{s}$, there is an integer $m$ such that $h$ is pseudo-isotopic to $\sigma^{m}$. So, showing this structure on the group of homeomorphisms of $X_{s}$ would solve Ingram's Conjecture.

In fact, there is a conjecture that could be used to show this property for the homeomorphisms of $X_{s}$.

The proof of Theorem 3 uses the fact that $\varphi:[0, \infty) \rightarrow X_{s}$ has a dense image. This motivated another look at the open arc $A_{0} \subset\left(I, f_{s}\right)$. It is dense in $\left(I, f_{s}\right)$ and a continuous one-to-one image of $[0, \infty)$. We found that we could carefully define a map $\varphi:[0, \infty) \rightarrow A_{0}$ so that if two homeomorphisms $h_{1}, h_{2}:[0, \infty) \rightarrow\left(I, f_{s}\right)$ have the property that there is an $M>0$ such that $\mid \varphi^{-1} \circ h_{1} \circ \varphi(x)-\varphi^{-1} \circ h_{2} \circ$ $\varphi(x) \mid<M$ for all $x$ in $[0, \infty)$, then $\left.h_{1}\right|_{X_{s}}$ and $\left.h_{2}\right|_{X_{s}}$ are pseudo-isotopic. For convenience we say that $h_{1}$ and $h_{2}$ are bounded $\bar{d}$-distance apart on $A_{0}$. Let us restate this result as a theorem.

Theorem 6 ([13]) Suppose that $h_{1}$ and $h_{2}$ are bounded $\bar{d}$-distance apart on $A_{0}$. Then $\left.h_{1}\right|_{X_{s}}$ and $\left.h_{2}\right|_{X_{s}}$ are pseudo-isotopic.

This leads to an approach which could solve Ingram's Conjecture in the general case.

Theorem 7 ([13]) Suppose that for every s and every homeomorphism $h:\left(I, f_{s}\right) \rightarrow\left(I, f_{s}\right)$ there is an integer $m$ such that $h$ and $\sigma^{m}$ are bounded $\bar{d}$-distance apart on $A_{0}$. Then Ingram's Conjecture holds.

\section{Concluding remarks.}

This paper has surveyed some recent results in the study of tent maps. There has been an effort to motivate the research and put it in perspective. Since the length of the paper is limited and the patience of the reader 
is likely also to be limited, the survey is not complete. It has concentrated mainly on results with which the author has been intimately involved. There are many more results that should have been mentioned to be comprehensive, but that would have required a book many times the length of this article.

However, there are a few more results that should be mentioned. The result of Kailhofer has been substantially improved. Stimac [38] has shown that if the forward orbit of $c$ is finite, then Ingram's Conjecture holds for those $s$. This, of course, includes the case that the forward orbit of $c$ is periodic. Another result has been announced by Raines and Stimac dealing with the case that the critical point $c$ is non-recurrent. However, the most difficult case still eludes us. For most values of $s$ in $(\sqrt{2}, 2)$, the orbit of $c$ will be dense in $[0,1]$. Almost nothing is known about Ingram's Conjecture in this case. It is a challenge not only to solve the conjecture in this case, but also to gain a clearer understanding of the structure of these inverse limits. It is probably best to leave the subject with this challenge in mind.

\section{Further reading}

For the interested reader, there is a considerable literature on one-dimensional dynamics. The book by Collet and Eckmann [17] is probably the best starting place. The article by Milnor and Thurston [34] was widely circulated before it was published and gives a good introduction to the kneading sequence for unimodal maps and its generalization to kneading matrices for general piecewise monotone maps. The book by de Milo and van Strien [33] brings one up to date at the time of writing in the early 1990s. Block and Coppel [11] was written with a different purpose in mind. It gives excellent coverage of the theory of topological entropy for maps of the interval. It is carefully written and is useful both for the beginner and the experienced researcher. The book by Brucks and Bruin [15] covers a wide range of topics from one-dimensional dynamics. It would be a good text for students preparing for research in the area.

\section{References}

[1] Abramov, L., (1959). The entropy of an automorphism of a solenoidal group, (Russian. English summary) Teor. Veroyatnost. i Primenen, 4, 249-254.

[2] Aarts, J. M. and Fokkink, R. J., (1991). The classification of solenoids, Proc. Amer. Math. Soc., 111, 1161-1163.

[3] Aarts, J. M. and Fokkink, R. J., (1998). Fixed points of the Bucket Handle, Proc. Amer. Math. Soc., 126, 881-885.

[4] Barge, M., (1986). Horseshoe maps and inverse limits, Pacific J. Math., 121, 29-39.

[5] Barge, M. and Diamond, B., (1995). Homeomorphisms of inverse limit spaces of one-dimensional maps, Fund. Math., 146, 2, 171-187.

[6] Barge, M., Brucks, K. and Diamond, B., (1996). Self-similarity in inverse limit spaces of the tent family, Proceedings of the American Mathematical Society, 124, 3563-3570.

[7] Barge, M. and Diamond, B., (1999). Subcontinua of the closure of the unstable manifod at a homoclinic tangency, Ergodic Theory Dynam. Syst., 19, 289-307.

[8] Barge, M. and Holte, S., (1995). Nearly one-dimensional Hénon attractors and inverse limits, Nonlinearity, 8, $29-42$.

[9] Bing, R. H., (1960). A simple closed curve is the only homogeneous bounded plane continuum that contains an arc, Canad. J. Math., 12, 209-230.

[10] Block, L., Keesling, J., Li, S-H. and Peterson, K., (1989). An improved algorithm for computing topological entropy, J. Statist. Phys., 55, 5-6, 929-939.

[11] Block, L. and Coppel, W., (1992). Dynamics in one dimension, Lecture Notes in Mathematics, 1513. SpringerVerlag, Berlin, viii+249 pp. 
[12] Block, L., Jakimovik, S., Kailhofer, L. and Keesling, J., (2005). On the classification of inverse limits of tent maps, Fundamenta Mathematicae, 187, 171-192.

[13] Block, L., Jakimovik, S. and Keesling, J., (2006). On Ingram's Conjecture, Topology Proceedings, 30, 95-114.

[14] Brucks, K. and Bruin, H., (1999). Subcontinua of inverse limit spaces of unimodal maps, Fund Math., 160, 219-246.

[15] Brucks, K. and Bruin, H., (2004). Topics from One-Dimensional Dynamics, London Mathematical Society, 297 pp.

[16] Bruin, H., (2000). Inverse limit spaces of post-critically finite tent maps, Fund. Math., 165, 125-138.

[17] Collet, P. and Eckmann, P.-P., (1980). Iterated Maps on the Interval as Dynamical Systems, Birkhauser, Boston.

[18] Guckenheimer, J. and Williams, R., (1979). Structural stability of Lorenz Attractors, Inst. Hautes Études Sci. Publ. Math., 50, 59-72.

[19] Guckenheimer, J. and Williams, R., (1979). The structure of Lorenz attractors, Inst. Hautes Études Sci. Publ. Math., 50, 73-99.

[20] Günther, B. and Segal, J., (1993). Every attractor of a flow on a manifold has the shape of a finite polyhedron, Proc. Amer. Math. Soc., 119, 321-329.

[21] Hénon, M. and Pomeau, Y., (1976). Two strange attractors with a simple structure, in Turbulence and NavierStokes equations, (Proc. Conf., Univ. Paris-Sud, Orsay, 1975), 29-68. Lecture Notes in Math. 565. [MR0448436 (56\#6742)]

[22] Hoffman, Karl, http://math.tulane.edu/ ₹khh/Lagniappe/dyadic_solenoid.png.

[23] Holte, S., (1992). Generalized horseshoe maps and inverse limits, Pacific J. Math., 156, 297-305.

[24] Ingram, W., (1995). Inverse limits on using tent maps and certain other piecewise linear bonding maps, Continua (Cincinnati, OH, 1994), 253-258, Lecture Notes in Pure and Appl. Math., 170, Dekker, New York.

[25] Juzvinskii, S., (1967). Calculation of the entropy of a group-endomorphism, (Russian) Sibirsk. Mat. \u Z., 8, 230-239.

[26] Kailhofer, L., (2003). A classification of inverse limit spaces of tent maps with periodic critical points, Fund. Math., 177, 2, 95-120.

[27] Kailhofer, L., (2002). A partial classification of inverse limit spaces of tent maps with periodic critical points, Topology Appl., 123, 2, 235-265.

[28] Keesling, J., (1972). The group of homeomorphisms of a solenoid, Trans. Amer. Math. Soc., 172, 119-131.

[29] Keesling, J. and Ssembatya, V., (2005). On fixed points of Knaster continua, Top. and Appl., 153, 318-326.

[30] Kwapisz, J., (2001). Homotopy and dynamics for homeomorphisms of solenoids and Knaster continua, Fund. Math., 168, 3, 251-278.

[31] Lorenz, E., (1963). Deterministic nonperiodic flow, J. Atmos. Sci., 20, 130-141.

[32] McCord, M. C., (1965). Inverse limit sequences with covering maps, Trans. Amer. Math. Soc., 114, 197-209.

[33] de Melo, W. and van Strien, S., (1993). One-Dimensional Dynamics, Springer, New York.

[34] Milnor, J. and Thurston, W., (1988). On iterated maps of the interval, in Dynamical systems, (College Park, MD, 1986-87), 465-563, Lecture Notes in Math., 1342, Springer, Berlin.

[35] Ruelle, D., (1976). The Lorenz attractor and the problem of turbulence, in Turbulence and Navier-Stokes equations, (Proc. Conf., Univ. Paris-Sud, Orsay, 1975),146-158. Lecture Notes in Math., 565, Springer, Berlin. 
[36] Scheffer, W., (1972). Maps between topological groups that are homotopic to homomorphisms, Proc. Amer. Math. Soc., 33, 562-567.

[37] Smale, S., (1967). Differentiable dynamical systems, Bull. Amer. Math. Soc., 73, 747-817.

[38] Stimac, S., (to appear). Classification of inverse spaces of tent maps with finite critical orbit, Top and Appl.

[39] Tucker, W., (1999). The Lorenz attractor exists, C. R. Acad. Sci. Paris Sér. I Math., 328, 1197-1202.

[40] Tucker, W., (2002). A rigorous ODE solver and Smale's 14th problem, Found. Comput. Math., 2, 53-117.

[41] Williams, R., (1967). One-dimensional non-wandering sets, Topology, 6, 473-487.

[42] Williams, R., (1974). Expanding attractors, Inst. Hautes Études Sci. Publ. Math., 43, 169-203.

[43] Williams, R., (1971). The structure of attractors, in Actes du Congrès International des Mathématiciens, (Nice, 1970), Tome 2,. 947-951. Gauthier-Villars, Paris.

[44] Williams, R., (1977). The structure of Lorenz attractors, in Turbulence Seminar, (Univ. Calif., Berkeley, Calif., 1976/1977), 94-112. Lecture Notes in Math., 615, Springer, Berlin, .

\author{
James Keesling \\ Department of Mathematics \\ University of Florida \\ Gainesville, FL 32611-8105 \\ U. S. A.
}

